\title{
Live-Vessel: Extending Livewire for Simultaneous Extraction of Optimal Medial and Boundary Paths in Vascular Images
}

\author{
Kelvin Poon ${ }^{1}$, Ghassan Hamarneh ${ }^{2}$, and Rafeef Abugharbieh ${ }^{1}$ \\ ${ }^{1}$ Biomedical Signal and Image Computing Lab, University of British Columbia \\ ${ }^{2}$ Medical Image Analysis Lab, Simon Fraser University, Canada
}

\begin{abstract}
This paper incorporates multiscale vesselness filtering into the Livewire framework to simultaneously compute optimal medial axes and boundaries in vascular images. To this end, we extend the existing 2D graph search to 3D space to optimize not only for spatial variables $(x, y)$, but also for radius values $r$ at each node. In addition, we minimize change for both scale and the smallest principle curvature and incorporate vessel boundary evidence in our optimization. When compared to two sets of DRIVE expert manual tracings, our proposed technique reduced the overall segmentation task time by $68.2 \%$, had a similarity ratio of 0.772 ( 0.775 between manual), and was $98.2 \%$ reproducible.
\end{abstract}

\section{Introduction}

Segmentation is vital for medical image analysis, but this task is difficult because biological structures typically exhibit significant variations due to subject diversity and pathology. In addition, imaging introduces artifacts, noise, and inter-camera variability. While automatic segmentation schemes are typically preferred, they still require human validation. On the other hand, manual tracing is often considered accurate, but is time consuming and may suffer from operator error and inter- and intra-operator variability [1. Due to these difficulties, semi-automated methods such as Livewire were introduced, offering high accuracy, efficiency, and reproducibility rates 2. Livewire utilizes sparsely spaced user-defined seedpoints to efficiently compute the optimal contour in between using Dijkstra's algorithm [3. Delineation accuracy can be increased in noisy regions at the expense of segmentation speed by providing a more dense set of seedpoints. However, for 2D images such as angiograms and retinal images, gradient-based Livewire implementations become inefficient due to overlapping vessels, complex branching networks, and low vessel contrast in thin vessels.

In previous work, angiography images were enhanced using 'vesselness' as an image feature 415]. These methods chose the 'optimal' scale $\sigma$ such that maximal vesselness response for individual pixels is achieved, but this selection can be highly influenced by noise. Sofka et al used multiscale matched filters to determine vessel medials, and used edge measures to affect the confidence of medial nodes [6]. However, they gave no consideration to the shape of the vessel and did not find an optimal medial. The problem of finding minimal paths in

N. Ayache, S. Ourselin, A. Maeder (Eds.): MICCAI 2007, Part II, LNCS 4792, pp. 444 451, 2007.

(C) Springer-Verlag Berlin Heidelberg 2007 
3D space was explored by Deschamps and Cohen [7], where a front propagation in $(x, y, z)$ was implemented, along with a method to approximate the medial axes of tubular structures using level sets. However, this work does not focus on identifying the boundaries of tubular structures. In [8, Young et al employed the 3D vesselness filter of Frangi et al [4] and used a front-propagation based algorithm to segment the vessel and extract the medial axis. However, because maximal vesselness response was chosen for each pixel prior to segmentation, this solution is not optimal. In the 'vessel crawlers' approach [9, locally optimal $\sigma$ was derived from the radius of the leading layer of the crawler. In [10], the locations and sphere radii of medial nodes were optimized to segment vascular structures. However, this method relied on near-uniform vessel intensities for optimization, and did not use vesselness features, which provide important magnitude and vessel direction information. In [11, Wink et al applied Dijkstra's algorithm to find the optimal medial and corresponding scale values based on vesselness. In this paper, we improve on their work by (i) adopting and extending the Livewire framework to compute the optimal path in $(x, y, r)$ space; (ii) including optimization costs to favour gradual change in both scale and the smallest principal curvature direction along the path to mitigate noise; and (iii) incorporating vessel boundary evidence from image pixels at a scale-dependent distance away from the medial as part of the optimal graph search. Furthermore, our method allows vessels to be segmentated with less seedpoints than traditional Livewire, and can simultaneously extract the medials and boundary points of vessels with radii down to 0.5 -pixel. The proposed technique was applied to the Digital Retinal Images for Vessel Extraction (DRIVE) archives [12. Our results were validated against manual segmentation.

\section{Method}

Our proposed framework is based on the original Livewire technique [2], where sparse seedpoints along an object boundary are input by a user and optimal contours connecting these points are found using Dijkstra's algorithm. This algorithm is a determinisic, exhaustive graph search that always finds the globally optimal path. Here, the medial axis of a blood vessel is defined as the optimal spatial coordinates $(x, y)$ and radius values $r$ between two points in $3 \mathrm{D}$ space $(x, y, r)$. This optimization is achieved by minimizing the cumulative cost function at each $(x, y, r)$ node. The incremental cost function, which describes the cost from node $q=(x, y, r)$ to a neighboring node $p=\left(x^{\prime}, y^{\prime}, r^{\prime}\right)$, is defined as:

$$
\operatorname{Cost}(q, p)=w_{1} C_{V}(p)+w_{2} C_{E v}(q, p)+w_{3} C_{I e}(p)+w_{4} C_{R}(q, p)+w_{5} C_{S}(q, p)
$$

$C_{V}$ is associated with Frangi's proposed multiscale vesselness filter [4], $C_{E v}$ refers to the vessel direction change between $q$ and $p$, and $C_{I e}$ is a measure of the fitness of a medial node by assessing the edge evidence of equidistant pixels on either side. Also, internal smoothness cost terms $\left(C_{R}\right.$ and $\left.C_{S}\right)$ are used to penalize paths in which the radius $r$ or the spatial variables $(x, y)$ fluctuate rapidly. These cost terms are explained in Section 2.2. and are normalized to lie in the range $[0,1]$. 


\subsection{Livewire Framework in $(x, y, r)$ Space: Live-Vessel}

We extend the original Livewire framework 2] to segment vascular structures. Rather than delineating a vessel by guiding the Livewire contour along boundaries, our method guides the Livewire along the vessel medial. This reduces the amount of seedpoints required, since a single optimal path defines three contours (the medial and two boundaries on either side). In 2D Livewire implementations, given an image $I(a)$, where $a=(x, y)$, the only local path choices from $a$ are to one of eight neighboring pixels $b=\left(x^{\prime}, y^{\prime}\right)$. In our proposed method, we optimize the medial with respect to three variables: the two spatial variables $x$ and $y$, and the vessel radius variable $r$. Accordingly, we extend the traditional Livewire graph search from $2 \mathrm{D}$ to $3 \mathrm{D}$. Since the medial axis in reality is $2 \mathrm{D}$ and cannot connect $q=\left(x, y, r_{i}\right)$ to $p=\left(x, y, r_{j}\right)(i \neq j$, i.e. only a single radius value can be associated with each medial node), our 3D graph search is restricted in this manner. To accommodate for vessels that dilate and constrict rapidly, the radius value can change to any other set of values (albeit penalized differently, as described in Section 2.2). This increases the computational complexity, but the optimal medial path and optimal vessel thickness are guaranteed.

Since the paths from the seedpoint to all nodes along the optimal path are also optimal, our proposed method only needs to record the location of the previous optimal node in its path. By following this node order, the optimal path from the seedpoint to any other point is quickly determined. To perform the actual segmentation, the medial path is first projected back onto the $x, y$ plane. Each medial node has an optimal radius value $r$, and its preceding and succeeding nodes form a direction vector. These elements are used to determine the two vessel boundary points on either side of the medial node. Repeating this for all medial nodes, the vessel is segmented.

\subsection{Live-Vessel External and Internal Costs}

The external cost terms in (1) are derived from the vessel filter $\left(C_{V}(p)\right)$ and from edge measures $\left(C_{I e}(p)\right)$. The internal cost terms are included to favour gradual changes in vessel direction $\left(C_{E v}(q, p)\right)$ and radius $\left(C_{R}(q, p)\right)$, as well as a spatially smooth medial $\left(C_{S}(q, p)\right)$.

Vesselness. To detect vascular structures in an image, we employ the multiscale vessel enhancement filter proposed by Frangi et al 4 for 2D images. The eigenvalues $\left|\lambda_{1}\right| \leq\left|\lambda_{2}\right|$ of the Hessian matrix $\mathcal{H}_{\sigma}$ of a Gaussian smoothed image $\left(I_{\sigma}=I * G\right.$, where $G$ has variance $\left.\sigma^{2}\right)$ are used to calculate vesselness as follows:

$$
C_{V}(q)=\mathcal{V}_{\sigma}\left(\lambda_{1}, \lambda_{2}\right)= \begin{cases}1 & \text { if } \lambda_{2}>0 \\ 1-\exp \left(-\frac{\mathcal{R}_{\mathcal{B}}^{2}}{2 \beta^{2}}\right)\left(1-\exp \left(-\frac{T^{2}}{2 c^{2}}\right)\right) & \text { otherwise }\end{cases}
$$

$\mathcal{R}_{\mathcal{B}}=\lambda_{1} / \lambda_{2}$ represents the eccentricity of a second order ellipse and $T=$ $\sqrt{\lambda_{1}^{2}+\lambda_{2}^{2}}$. $\beta$ and $c$ affect filter sensitivity and have values 0.5 and 0.3 respectively. These values are similar to those used in other vessel filter studies [411. 


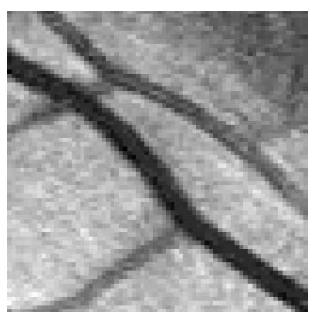

(a)

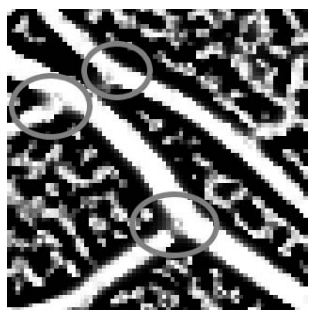

(b)

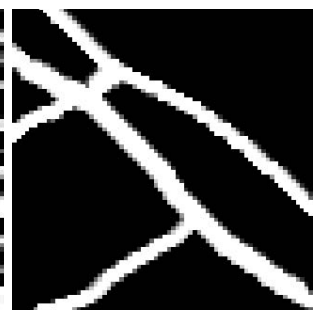

(c)

Fig. 1. (a) Original retinal image, cropped and magnified for clarity. (b) Maximal response vesselness filter output, scaled for contrast. Note the erroneous values at areas of bifurcation, vessel overlap, and high levels of noise. (c) Segmentation result with our proposed method.

While choosing the highest filter response for each pixel gives an estimated vessel size at each pixel location, this method however is susceptible to noise and does not enforce spatial continuity (Figure 1(b)). Our proposed method, in contrast, stores the results over a range of scales separately 1 . By combining these results with other cost factors, we place restrictions on the relationship of neighboring nodes; hence, the proposed method is robust to noise and poor image quality (Figure 1(c)).

Vessel Direction Consistency. The eigenvector $E v(x, y, r)$ of $\mathcal{H}_{\sigma}$, corresponding to $\lambda_{1}$, points in the direction of the minimum principal curvature, which estimates the vessel direction [4]. By choosing paths that minimize the change in direction of $E v$, we can mitigate local noise that occurs in vascular images. We therefore incorporate the cost term $C_{E v}(q, p)$ from (11) and define it as:

$$
C_{E v}(q, p)=\frac{2}{\pi} \arccos \left|\frac{E v(p) \cdot E v(q)}{|E v(p)||E v(q)|}\right|,
$$

where $q=\left(x, y, \sigma_{1}\right), p=\left(x^{\prime}, y^{\prime}, \sigma_{2}\right)$, are node coordinates. Since $E v(x, y, r)$ points arbitrarily in either directions of a bidirectional vessel $\left( \pm 180^{\circ}\right)$, we take the absolute value in (3) to obtain the smaller angle between the vectors.

Image Evidence Using Edge Detection. Our proposed method also uses edge evidence to favour medial nodes that are located at the centre of a vessel cross-section with radius $r$. To compute this, we employ gradient, Canny, and Laplacian of Gaussian (LoG) edge detection to estimate vessel boundaries and average their responses into $R(x, y)$. We chose these filters because Canny and LoG filters are less sensitive to noise, whereas the gradient magnitude filter does not involve pre-smoothing thus complementing the Canny and LoG filters by detecting weak structural edges.

\footnotetext{
${ }^{1}$ Defining boundaries as zero crossings of the Laplacian of a Gaussian intensity profile across the vessel yields $\sigma=r$, where $r=$ vessel radius. This is derived by equating the second derivative of the Gaussian kernel to zero.
} 


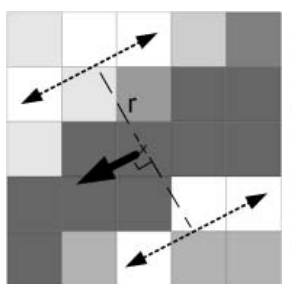

(a)

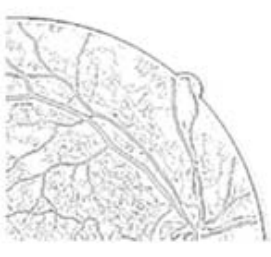

(b)

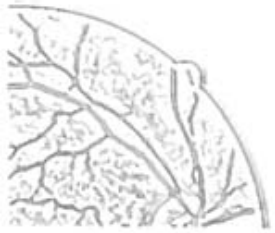

(c)

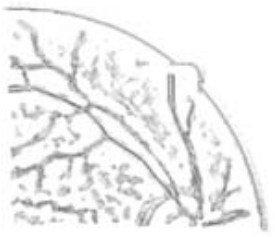

(d)

Fig. 2. (a) A node's vessel direction is denoted by the thick arrow, and the evenly spaced points along the bidirectional arrows are tested for edge detection response. (b) Image evidence cost function $C_{S}(q, p)$ of a retinal image at radius $r=1$. Medial nodes of vessels with this radius exhibit the lowest cost. Larger vessels' edges are faintly detected, but their medials are not. (c)-(d) Cost function $C_{S}(q, p)$ of the same retinal image at $r=3,4$ respectively. Medials of larger vessels exhibit the lowest cost. Medials of smaller vessels are no longer detected.

For each $q=(x, y, r)$ node in the image, we combine the vesselness direction $E v(x, y, r)$ and $R(x, y)$ to define another measure of node 'medialness'. By finding the unit vector that is normal to $E v$ in the $(x, y)$ plane and scaling it by $r$, we can determine two locations at which the vessel wall should be located. Then, our method retrieves the corresponding $R(x, y)$ at these points and at adjacent points $P_{R}=\left\{\left(x_{i}, y_{i}\right) ; i=1,2 \ldots N\right\}$ ( $N$ total points to analyze, including both sides $)$ in parallel directions to $E v$ (Figure 2a). The image evidence cost $C_{I e}(q)$ in (1) is then defined as $C_{I e}(q)=1-(1 / n) \sum_{i=1}^{N} R\left(P_{R}\right)$ to average a potentially noisy response. This cost term is minimized for a medial node that has equidistant (distance $=r$ ) vessel boundaries, which is expected for vessel medial nodes.

Spatial and Radius Smoothness Constraints. Our proposed approach also imposes a small constant value cost for each additional pixel added to the path (connecting points $q=(x, y, r)$ to $p=\left(x^{\prime}, y^{\prime}, r^{\prime}\right)$ ), which accumulates during the graph search operation. This cost, $C_{S}(q, p)$ in (11), is proportional to $\sqrt{\left(x-x^{\prime}\right)^{2}+\left(y-y^{\prime}\right)^{2}}$. By encouraging the medial axis to be shorter, contour jaggedness is avoided.

Similarly, we penalize medial paths with rapidly changing radius values for two reasons. Firstly, the vesselness filter is noise sensitive, and estimating the radius based solely on the filter output is unreliable. Secondly, vessel radii tend to not change rapidly unless branching occurs. By adding this cost $C_{R}(q, p)=$ $\left|r-r^{\prime}\right| /\left(r_{\max }-r_{\min }\right)$ to (1) , the vessel width in the segmentation result is rendered smoother with gradually changing radius values. Here, $r_{\max }$ and $r_{\min }$ are the maximum and minimum values $r$ can take.

\section{Results and Discussion}

Our proposed method was validated by segmenting images from the DRIVE database [12. To demonstrate its capabilities, our method's performance 


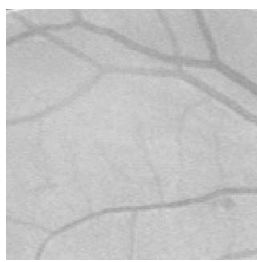

(a)

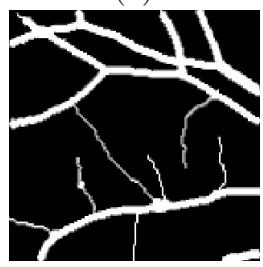

(d)

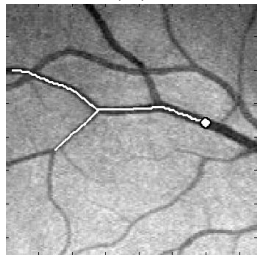

(g)

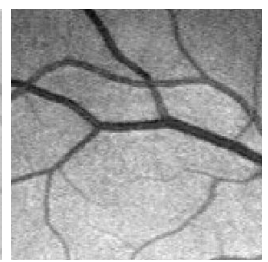

(b)

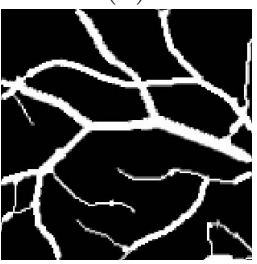

(e)

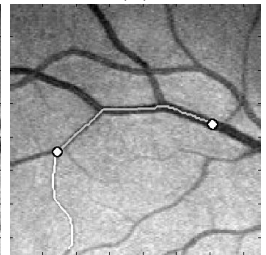

(h)

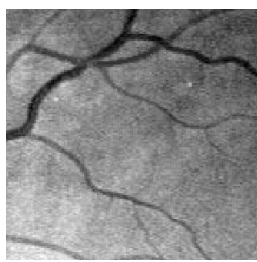

(c)

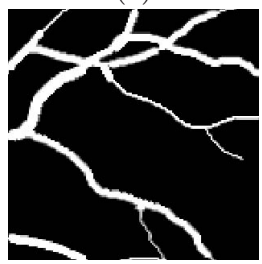

(f)

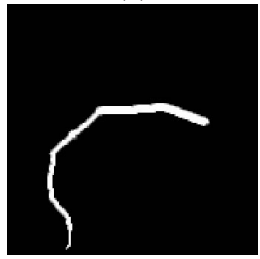

(i)

Fig. 3. (a)-(c) Original images, cropped for illustrative purposes. (d)-(f) Segmentation masks using Live-Vessel. (g) 'Live' aspect of Live-Vessel. A seedpoint is input by the user (circle), and optimal paths depending on the cursor location are displayed (two shown). (h) Another seedpoint locks the first segment, and this process is repeated until the vessel is segmented. (i) Resulting mask from using 3 seedpoints.

during these tasks was quantified based on three recommended criteria for semiautomatic segmentation [13]: reproducibility, accuracy, and efficiency. Sensitivity of our method to parameter setting was also analyzed. We were able to segment vessels of varying widths, down to one pixel-wide vessels (radius $r=0.5$ ). Qualitative result examples (showing $140 \times 140$ pixels from the original $565 \times 584$ images) are illustrated in Figure 3 .

Reproducibility was measured by performing several segmentation trials of the same task, which differed in seedpoint selection. Since reproducibility does not compare segmentations to an absolute truth, we subjected the results of these trials to pairwise Dice similarity tests. We found the reproducibility rate to be 0.987, 0.979, and 0.982 for our test images (Figure 3). These rates are high and consistent with previous Livewire validations, reflecting that the locations of sparse seedpoints do not greatly impact the optimal contour in between.

Accuracy of a segmentation is normally quantified by analyzing the percentage of false positives and false negatives of a segmentation compared to the golden truth. However, in retinal vessel segmentation, blood vessel boundaries are oftentimes unclear and thin vessels are perceived differently by different observers. 
Table 1. Summary of the average accuracy and efficiency results of our proposed Live-Vessel compared to manual traces (image size of $565 \times 584$ pixels). Accuracy was measured as a Dice similiarity to each image's two DRIVE expert segmentations. Efficiency was measured as the reduction in manual task time needed to generate the mask. Results shown are averages over a span of three trials.

\begin{tabular}{|c|c|c|c|}
\hline \multicolumn{4}{|c|}{ Segmentation Accuracy } \\
\hline & Trace1 vs Trace2 & Live-Vessel vs Trace1 & Live-Vessel vs Trace2 \\
\hline Image1 & 0.762 & 0.769 & 0.762 \\
\hline Image2 & 0.766 & 0.773 & 0.791 \\
\hline Image3 & 0.799 & 0.769 & 0.772 \\
\hline \multicolumn{3}{|c|}{ Segmentation Efficiency } \\
\hline & Tracing (hh:mm:ss) & Live-Vessel (hh:mm:ss) & Time reduction (\%) \\
\hline Image1 & $01: 47: 32$ & $00: 31: 43$ & 70.3 \\
\hline Image2 & $01: 53: 00$ & $00: 33: 58$ & 69.9 \\
\hline Image3 & $01: 28: 12$ & $00: 31: 12$ & 64.5 \\
\hline
\end{tabular}

Therefore, we calculated the similarity to each manual tracing and compared that to how similar the manual tracings are to each other (Table 1). We found our results to be similar to both manual segmentations and in one case, even exceeded the similarity between the manual tracings themselves.

Efficiency was measured as the reduction in the manual segmentation task time when using our proposed method (Table 1). We manually segmentated each image using a simple paint tool, and the task times were similar to those reported in [12]. As expected, Live-Vessel, similar to Livewire, drastically reduced the manual task time required.

Sensitivity analysis was done to determine the change in accuracy while varying each weight value. Our implementation by default uses equal weighting $\left(w_{1 . .5}=1\right)$ for each term in (11). We found that varying each weight by $\pm 50 \%$ did not change the accuracy by more than $5.2 \%$ for our test images.

\section{Conclusions}

This paper introduced a novel semi-automated method for segmentation of vascular images. The method incorporates multiscale vesselness filtering into the conventional Livewire framework to efficiently compute optimal medial axes. Along a medial, we simultaneously optimize for the spatial variables $(x, y)$ as well as the vessel thickness variable $r$, extending the traditional 2D graph search to $3 \mathrm{D}(x, y, r)$. In addition, we also optimize for vessel direction from smallest principle curvature direction and boundary evidence at a scale-dependent distance away from the boundary. When applied to 2D retinal images, our proposed method had a high reproducibility rate and significantly reduced segmentation task time compared to manual tracing. Also, accuracy was comparable to manual tracing. Our future work will focus on reducing the computation time required for the 3D graph search to increase efficiency. By reducing computation time, it 
will enable our method to be extended even further to $(x, y, z, r)$ for $3 \mathrm{D}$ vessel segmentation and still support user-interaction. Also, automatic determination of suitable seedpoint candidates along the medial is currently being investigated.

\section{References}

1. Rajapakse, J., Kruggel, F.: Segmentation of MR images with intensity inhomogeneities. Image and Vision Computing 16, 165-180 (1998)

2. Barrett, W., Mortensen, E.: Interactive live-wire boundary extraction. Medical Image Analysis 1, 331-341 (1997)

3. Dijkstra, E.: A note on two problems in connexion with graphs. Numerical Mathematik 1, 269-270 (1959)

4. Frangi, A., Niessen, W., Vincken, K., Viergever, M.: Multiscale vessel enhancement filtering. In: Wells, W.M., Colchester, A.C.F., Delp, S.L. (eds.) MICCAI 1998. LNCS, vol. 1496, pp. 130-137. Springer, Heidelberg (1998)

5. Krissian, K., Malandain, G., Ayache, N., Vaillant, R., Trousset, Y.: Model based multiscale detection of 3D vessels. In: IEEE CVPR, pp. 722-727. IEEE Computer Society Press, Los Alamitos (1998)

6. Sofka, M., Stewart, C.: Retinal vessel centerline extraction using multiscale matched filters, confidence and edge measures. IEEE TMI 25, 1531-1546 (2006)

7. Deschamps, T., Cohen, L.: Fast extraction of minimal paths in 3D images and applications to virtual endoscopy. Medical Image Analysis 5, 281-299 (2001)

8. Young, S., Movassaghi, B., Weese, J., Rasche, V.: 3D vessel axis extraction using 2D calibrated x-ray projections for coronary modeling. SPIE Medical Imaging, 1491-1498 (2003)

9. McIntosh, C., Hamarneh, G.: Vessel crawlers: 3D physically-based deformable organisms for vasculature segmentation and analysis. In: IEEE CVPR, pp. 10841091. IEEE Computer Society Press, Los Alamitos (2006)

10. Li, H., Yezzi, A.: Vessels as 4D curves: Global minimal 4D paths to 3D tubular structure extraction. In: IEEE Computer Society Workshop on MMBIA, IEEE Computer Society Press, Los Alamitos (2006)

11. Wink, O., Niessen, W., Viergever, M.: Multiscale vessel tracking. IEEE TMI 23, 130-133 (2004)

12. Niemeijer, M., Staal, J., van Ginneken, B., Loog, M., Abramoff, M.: Comparative study of retinal vessel segmentation methods on a new publicly available database. SPIE Medical Imaging, 648-656 (2004)

13. Olabarriaga, S., Smeulders, A.: Interaction in the segmentation of medical images: A survey. Medical Image Analysis 5, 127-142 (2001) 